\title{
PROTECCIÓN DE DATOS DE PRUEBA Y SU EXCLUSIVIDAD EN MEDICAMENTOS Y AGROQUÍMICOS: LA INTERPRETACIÓN DEL ARTICULO 39.3 ADPIC
}

\author{
PROTECTION OF TEST DATA AND EXCLUSIVITY IN MEDICINES AND \\ AGROCHEMICALS: THE INTERPRETATION OF ARTICLE 39.3 TRIPS

\begin{abstract}
PROTEÇÃO DE DADOS DE PROVA E SUA EXCLUSIVIDADE EM MEDICAMENTOS E AGROQUÍMICOS: A INTERPRETAÇÃO DO ARTIGO 39.3
\end{abstract}

\author{
Marta Carolina Gimenez Pereira \\ http://orcid.org/0000-0001-5661-8860 / http://lattes.cnpq.br/6905306640861147 / magipe@hotmail.com \\ Universidade Federal da Bahia, UFBA. \\ Salvador, BA, Brasil.
}

\begin{abstract}
RESUMO
As novas tendências em Propriedade Intelectual nos remetem ao Acordo TRIPS ao analisar a "informação não divulgada" e nela a "exclusividade de dados" prevista no artigo 39.3. No entanto, existem interpretações difíceis para esta figura de controvérsia que não escapa aos interesses dos diferentes setores participantes dos mais variados membros da OMC. O presente estudo visa abordar a figura a partir de seus conceitos e requisitos, analisando suas diversas interpretações, bem como as sensibilidades em sua aplicação. O método utilizado é o dedutivo e a técnica de pesquisa bibliográfica. Está estruturado em introdução, desenvolvimento, conclusões e bibliografia.
\end{abstract}

Palavras-chave: agrotóxicos; Artigo 39.3 TRIPS; dados de teste; medicamentos; propriedade intelectual.

\section{ABSTRACT}

The new trends in Intellectual Property refer us to the TRIPS Agreement when analyzing the "undisclosed information" and in it the "data exclusivity" provided in article 39.3. However, there are difficult interpretations for this controversial figure that does not escape the interests of the different participating sectors of the most varied members of the WTO. The present study aims to approach the figure from its concepts and requirements, analyzing its various interpretations as well as the sensitivities in its application. The method used is the deductive and the bibliographic research technique. It is structured in introduction, development, conclusions and bibliography.

Keywords: agrochemicals; Article 39.3 TRIPS; intellectual property; medicines; test data.

\section{RESUMEN}

Las nuevas tendencias en Propiedad Intelectual nos remiten al ADPIC al analizar la "información no divulgada" y en ella la "exclusividad de datos" prevista en su artículo 39.3. Empero, existen difíciles interpretaciones para esta polémica figura que no escapa a los intereses de los distintos sectores participantes de los más variados miembros de la OMC. El presente estudio tiene por objeto abordar la figura desde sus conceptos y requisitos, analizando sus variadas interpretaciones así como las sensibilidades en su aplicación. El método utilizado es el deductivo y la técnica de investigación bibliográfica. Se estructura en introducción, desarrollo, conclusiones y bibliografia.

Palabras clave: agroquímicos; artículo 39.3 ADPIC; datos de prueba; medicamentos; propiedad intellectual. 


\section{SUMÁRIO}

INTRODUCCIÓN; 1 CONCEPTUALIZACION, NORMATIVIDAD INTERNACIONAL E INTERPRETACIÓN DE LA PROTECCIÓN DE DATOS DE PRUEBA: EL INICIO DE LA POLÉMICA. LA DOCTRINA DE LA JUSTA RETRIBUCIÓN; 2 IMPLICANCIAS DE ADPIC PLUS EN LA FIGURA DE LA EXCLUSIVIDAD DE DATOS; CONCLUSIÓN; BIBLIOGRAFÍA.

\section{INTRODUCCÍON}

La protección de datos de prueba se asemeja a la noción de exclusividad de datos que los norteamericanos y la Comisión Europea adoptaron en 1984 y 1986 respectivamente. Así, nociones de 'protección de datos de prueba', 'exclusividad de datos' e 'información confidencial protegida' son conceptos afines, aunque esta última es más bien una noción dentro de la anterior denominación que fue adoptada por Estados Unidos ya desde la época de la mencionada Ronda Uruguay.

En el concepto norteamericano hay que puntualizar y subrayar que no sólo se pretendía prohibir en la época la utilización de los datos objeto de las pruebas de productos farmacéuticos y agroquímicos sino también se buscaba prohibir basarse en ellos, iniciativa que no prosperó (CORREA, 2014, p. 14). Siendo enfáticos reiteramos que la idea era doble: constreñir la utilización de los datos, así como basarse en ellos.

El artículo quedó redactado bajo el número 39.3 del Acuerdo sobre los Aspectos de los Derechos de Propiedad Intelectual relacionados con el Comercio, en adelante ADPIC, como sigue: "Los Miembros, cuando exijan, como condición para aprobar la comercialización de productos farmacéuticos o de productos químicos agrícolas que utilizan nuevas entidades químicas, la presentación de datos de pruebas u otros no divulgados cuya elaboración suponga un esfuerzo considerable, protegerán esos datos contra todo uso comercial desleal. Además, los Miembros protegerán esos datos contra toda divulgación, excepto cuando sea necesario para proteger al público, o salvo que se adopten medidas para garantizar la protección de los datos contra todo uso comercial desleal". Sin embargo, el problema radica en que son muchas las discusiones en cuanto a la interpretación y alcance del artículo previsto en ADPIC y que exige la protección de los datos de prueba sobre la seguridad y eficacia de productos farmacéuticos y 
agroquímicos, bajo la disciplina de la competencia desleal y las normas del derecho internacional por verse prevista en un Acuerdo marco.

El artículo tiene por objetivo abordar la figura desde sus conceptos y requisitos, analizando sus variadas interpretaciones, así como las sensibilidades que originan los problemas en su aplicación, para lo cual utilizará una metodología deductiva y la técnica de investigación bibliográfica.

Se desarrollarán dos tópicos. El primero de ellos conceptualiza la figura, la pone en contexto en la normativa internacional y aborda la interpretación de la noción de protección de datos de prueba, encarando la polémica iniciada y sugiriendo la doctrina de la justa retribución como solución. Luego, se describen las implicancias del futuro de ADPIC, a saber, ADPIC PLUS, en la exclusividad de datos. Finalmente se presentan las conclusiones y la bibliografía.

\section{CONCEPTUALIZACION, NORMATIVIDAD INTERNACIONAL E} INTERPRETACIÓN DE LA PROTECCIÓN DE DATOS DE PRUEBA: EL INICIO DE LA POLÉMICA. LA DOCTRINA DE LA JUSTA RETRIBUCIÓN

Es el artículo 39.3 del ADPIC de 1994, el que introduce la regulación internacional, de forma vinculante, sobre lo que se conoce como "información no divulgada" y la protección de los datos de prueba sobre la seguridad y eficacia de productos farmacéuticos y agroquímicos. Veamos primeramente qué comprende.

Correa nos dice que según ADPIC la noción cubre dos categorías diferentes de información: la que se conoce como "secretos comerciales" y la que nos ocupa, que son los "datos de prueba". Los datos de prueba se definen como los resultados de ensayos clínicos que se llevan a cabo para demostrar la seguridad y eficacia de productos farmacéuticos y agroquímicos y según el autor su protección fue unos de los temas que consiguió dividir el norte y el sur durante las negociaciones de la Ronda Uruguay, en su momento ${ }^{1}$.

\footnotetext{
${ }^{1}$ CORREA, Carlos. Protección de datos de prueba: Derechos conferidos bajo el Acuerdo sobre los ADPIC y algunos efectos de los estándares ADPIC-PLUS en Acceso a los medicamentos e información confidencial. Buenos Aires: Ciudad Argentina-Hispania libros, 2014, pp. 13 y 14.
} 
La protección consiste en la prohibición de utilización de tales datos para obtener la aprobación de comercialización de un producto farmacéutico o agroquímico. Consiste en un derecho de protección que ostenta el titular de los datos contra prácticas comerciales desleales, evitándose expresamente el uso o la divulgación, salvo casos específicos. Conlleva ese derecho una obligación de no hacer, obligación negativa o de abstención, a más de una obligación de protección consecuente.

Se trata de una prerrogativa otorgada por los países miembros de la Organización Mundial de Comercio, en adelante OMC, que obliga a proteger los datos de prueba llevados a cabo por el titular contra prácticas de competencia desleal, siendo esta disciplina la que regula la figura de la información no divulgada expresada más arriba, según surge del artículo 10 bis del Convenio de París. ${ }^{2}$

Diferentes interpretaciones se han dado a raíz de esta redacción del artículo 39.3, tal como en la práctica ocurrió con diversos acuerdos bilaterales y multilaterales celebrados entre países miembros de la OMC. El principal argumento en contra de esta protección la encuentran una vez más las patentes de medicamentos y los detractores se sostienen con fuerza en los efectos que la norma acarrea señalando que si el gobierno utiliza los datos de prueba para aprobar versiones genéricas de medicamentos no existe uso desleal ${ }^{3}$ y añaden que tal disposición de ADPIC no debería conllevar en automático una concesión de exclusividad ni remuneración.

Un sensible punto se presenta sobre estos matices de esos efectos: la intervención del gobierno en la entrada de los medicamentos genéricos como parte de sus políticas públicas así como el impedimento de que la normativa citada de ADPIC se constituyen en una suerte de trampolín para acceder a mayores derechos que los que confiere una patente, tentando

\footnotetext{
2 Este artículo 10 bis del Convenio de París dispone: “Competencia desleal: 1) Los países de la Unión están obligados a asegurar a los nacionales de los países de la Unión una protección eficaz contra la competencia desleal. 2) Constituye acto de competencia desleal todo acto de competencia contrario a los usos honestos en materia industrial o comercial. 3) En particular deberán prohibirse: i) cualquier acto capaz de crear una confusión, por cualquier medio que sea, respecto del establecimiento, los productos o la actividad industrial o comercial de un competidor; ii) las aseveraciones falsas, en el ejercicio del comercio, capaces de desacreditar el establecimiento, los productos o la actividad industrial o comercial de un competidor; iii) las indicaciones o aseveraciones cuyo empleo, en el ejercicio del comercio, pudieren inducir al público a error sobre la naturaleza, el modo de fabricación, las características, la aptitud en el empleo o la cantidad de los productos".

3 El uso de tales datos por parte del gobierno a través de sus entidades a fin de estudiar aplicaciones subsiguientes con miras a que obtengan la aprobación de comercialización no puede considerarse como un "uso comercial".
} 
beneficiarse de exclusividades y regalías bajo el argumento de la debatida figura de "data protection", como se conoce en inglés.

Estados Unidos y la Unión Europea argumentan que el mencionado artículo 39.3 de ADPIC ordena la protección de datos y su exclusividad para el titular. Sin embargo, el sentido ordinario de la terminología empleada en ese artículo, interpretado en el contexto de la terminología de “competencia desleal” en el Convenio de París y más aún en la negociación histórica del artículo 39.3, pone en duda este punto de vista ${ }^{4}$.

La polémica del siempre pretender más de una norma o ir más allá de la norma es esa espina que hinca, desatada por Estados Unidos ya en aquel entonces, que no ha encontrado un punto de detenimiento y que se refleja incluso en el denominado ADPIC PLUS así como en el Acuerdo Transpacífico o TPP por su siglas en inglés, a la fecha de hoy no muerto sino latente a pesar de la postura política norteamericana de 2017 que parecía acabar con él, análisis que no corresponde a este estudio pero que resulta pertinente mencionar a modo ilustrativo.

Otra de las problemáticas que rondan en cuanto a la interpretación del artículo en comento se refiere a qué tipo de protección deben dar los países partes de la OMC a esta figura, es decir, al alcance de la norma a la hora de celebrar los Tratados de Libre Comercio.

Por un lado, la doctrina ha estudiado literalmente el artículo 39.3 y de la opinión académica se colige que bastaría con circunscribir la figura a la disciplina de la competencia desleal, la cual no exige el reconocimiento ni la aplicación de derechos exclusivos, añadiendo que más bien el otorgamiento de la exclusividad constituiría una derogación drástica del principio de libre competencia ${ }^{5}$. De hecho, el ADPIC contiene suficientes flexibilidades para que un país determine los medios adecuados para proteger los datos de prueba ${ }^{6}$. El mismo párrafo 4 de la Declaración de Doha señala que las disposiciones “...deben ser interpretadas y aplicadas de manera tal que se avale el derecho de los Miembros de la OMC de proteger la salud pública y, en

\footnotetext{
${ }^{4}$ BASHEER, Shamnad. Protection of Regulatory Data Under Article 39.3 of Trips: The Indian Context. Intelectual Property Institute Journal, 2006, p. 11. Disponible en: https://ssrn.com/abstract=934269. Consultado el: 7 may. 2018.

${ }^{5}$ En tal sentido autores como Carlos Correa, Charles Clift, Lucas Arrivillaga, Jean-Frédéric Morin, Jerome Reichman, Pedro Roffe, Christoph Spennemann, citados por Correa.

${ }^{6}$ De acuerdo con el Acuerdo ADPIC, si la autoridad regulatoria de un país solicita la presentación de datos no divulgados al titular para el registro del medicamento o agroquímico, la exigencia para el país miembro de la OMC no es sino la de proteger esos datos contra su uso comercial desleal en caso de tratarse de una nueva entidad química y en caso de que la elaboración de tales datos haya supuesto un "esfuerzo considerable". Más adelante se apuntará la postura que el concepto abarcaría el de "uso comercial" de forma amplia y no tan sólo el "uso comercial desleal".
} 
particular, de promover el acceso a los medicamentos para todos" y por lo tanto debería adoptarse la postura de usarse las flexibilidades de ADPIC teniendo en cuenta el interés nacional del país de que se trate ${ }^{7}$, lo cual parece ser lo más equilibrado.

Por otro lado, si la norma internacional establece la obligación de exclusividad, deja a los países miembros la tarea de determinar la duración de tal obligación con el consecuente riesgo de que alguno de ellos otorgue tal protección por un tiempo ilimitado a su titular lo que vendría a acentuar la brecha entre los países industrializados y aquellos en vías de desarrollo en cuanto a la discusión de la pertinencia de la protección o inclusive entre aquellos o éstos mismos en lo que respecta a la duración del período de exclusividad.

Hay que tener el cuidado en apuntar que los países en desarrollo han sentado la postura en este sentido, negándose en su mayoría a conceder la protección ${ }^{8}$ : el artículo 39.3 ADPIC deja un espacio considerable a los países miembros para implementar la obligación de proteger datos de prueba; la información no divulgada se encuentra ya regulada por la competencia desleal en el artículo 10 bis del Convenio de París; la información no divulgada no debe ser abordada dentro del concepto de "propiedad" y no exige conceder derechos exclusivos al titular de los datos 9 .

Los detractores de la protección en comento han sustentado su razonamiento en que la investigación y desarrollo ( $I+D)$ de nuevos productos farmacéuticos y agroquímicos es impulsada fundamentalmente por países desarrollados con lo cual la figura de exclusividad de datos no debería aplicarse en países en desarrollo por la razón de que siendo que los desarrollados representan el $85 \%$ del mercado mundial de medicamentos bajo receta de productos farmacéuticos, es improbable que la falta de esa exclusividad en uno o más países en desarrollo sea determinante para una empresa a la hora de decidir invertir en producir los datos de prueba.

\footnotetext{
7 SATWANT Reddy y GURDIAL Singh Sandhu. Report on Steps to be taken by Government of India in the context of Data Protection Provisions of Article 39.3 of TRIPS Agreement o Informe de India, p. 4. Disponible en: https://www.indiaoppi.com/sites/default/files/PDF\%20files/Data\%20Protection\% 20report.PDF._Consultado el: 7 may. 2018.

8 Tendencia que en los últimos años se vio disminuida en razón de la presión que ejerce la OMC, la firma de un Tratado de Libre Comercio o inclusive por presiones unilaterales. Entre los países que la han rechazado se citan Argentina e India. En el caso de Colombia, al haber suscrito diversos Tratados de Libre Comercio con Estados Unidos, la Asociación Europea de Libre Comercio (AELC) y con la Unión Europea, se suma al compromiso de otorgar protección a los datos de prueba concediendo períodos de exclusividad. Canadá es otro de los países que se cita entre los que han otorgan el período de exclusividad de datos. Inclusive lo han ampliado de cinco a ocho años, más seis meses de exclusividad pediátrica, según las enmiendas del año 2006 hechas al Reglamento Canadiense sobre Alimentos y Fármacos.

${ }_{9}^{9}$ Postura sentada en reunión del Consejo del Acuerdo sobre los ADPIC.
} 
Por otro lado, sostiene que el tiempo de ventaja que obtiene la compañía que origina tales datos, así como la misma figura de la patente como vía de protección, resultan suficientes para recuperar los costos del desarrollo de tales datos ${ }^{10}$.

Sin embargo, esto es discutible porque la intención primigenia de la norma es precisamente la de ofrecer oportunidades comerciales más justas a los titulares de datos y sobre todo armonizar un diseño de políticas efectivas con legítimos reclamos de derechos exclusivos limitados por el interés público y que se puedan defender en términos del bienestar público real ${ }^{11}$, promoviendo la necesidad de aumentar las actividades de innovación e I+D en productos farmacéuticos también en países en vías de desarrollo o aquellos que son productores de principios activos de productos farmacéuticos y agroquímicos, como el caso de India, en forma adecuada y utilizando tanto el rico capital humano existente a nivel local como su infraestructura $^{12}$. En esta armonización se incluye el incentivo de lograr el progreso de los países en vías de desarrollo. El argumento de que hoy en día la mayoría de los productos farmacéuticos y agroquímicos son elaborados por países desarrollados, teniendo una posición de mercado dominante, se encontraría en cierta forma limitado precisamente por el reclamo de mayor presencia de I+D en aquellos países, sin desdeñar la realidad propia que los incumbe y las propias diferencias entre tales países en vías de desarrollo. El ejemplo es la India como productor importante de principios activos.

Aun así, en las negociaciones del mencionado artículo 39.3, las partes en su mayoría rechazaron la propuesta norteamericana eligiendo más bien hacer hincapié a la aplicación de las reglas de competencia desleal el cual no implica el reconocimiento de derechos exclusivos. En la disputa de introducción de la exclusividad de datos, como se ha mencionado, sea a través de modificaciones a la legislación nacional, de negociaciones de Tratados de Libre Comercio o de acuerdos bilaterales, así como a través de los procesos de adhesión a la OMC de nuevos integrantes, se ha notado que la representación de la industria de productos de marca o big pharma ha apoyado activamente la introducción de cinco o más años de exclusividad en tanto

\footnotetext{
${ }^{10}$ CORREA, op. cit., pp. 20 y 21.

11 Como expresa Antony Taubman, actual Director de la División de Propiedad Intelectual de la OMC, que propone una lectura heterodoxa del artículo 39.3, basándose en una interpretación de relación justa entre competidores y el cumplimiento de las expectativas de negociaciones de los países sobre el acceso a la protección de la PI.

${ }^{12}$ Cfr. op. cit., informe de la Declaración Ministerial de Doha en Doha WTO Ministerial 2001 del 20 noviembre 2001, WT/MIN(01)/DEC/1, disponible en http://www.wto.org/english/thewto_e/minist_e/min01_e/mindecl_e.htm, consultada el 15 de marzo de 2018.
} 
que el segmento genérico de la industria farmacéutica ha disentido enérgicamente con esta postura $^{13}$.

Según se anticipó, otros matices tienen que ver con la diferencia entre "basarse" y "utilizar" amén de que dependerá de quién sea quien los utiliza pues, como se ha visto, el gobierno, para cumplir su tarea a través de autoridades, precisa usar esos datos a fin de evaluar aplicaciones futuras que procurarán la aprobación de la comercialización, lo cual no implica un uso "comercial" de tales datos. Además, hablando de una posible ventaja comercial que puedan tener los competidores con tal uso, ella es totalmente lícita si no conlleva prácticas deshonestas. $Y$ es que, hasta en esta última palabra cabrían sutilezas si consideramos que lo “desleal” y lo "deshonesto" dependerá de la cultura que se analice.

Podemos concluir en este tópico que la obligación que tienen los países parte conlleva una protección con libertad de determinar la forma de proteger esos datos. La obligación supone:

1) No obtener datos de prueba no divulgados por medio de prácticas deshonestas.

2) No dar a los datos los datos de prueba un uso comercial.

Siempre en cuanto a la interpretación de la norma, pero añadiendo la justa retribución por el uso de los datos de prueba, autores sostienen que la figura puede ser admitida toda vez que se garantice una remuneración al titular por su uso, cumplidos los dos presupuestos básicos que son: que los datos de las entidades farmacéuticas o agroquímicas no hayan sido divulgados y que su producción implique un esfuerzo considerable.

Afirma BASHEER, al hacer el estudio del caso de la India, que el estándar mínimo que impone el artículo 39.3 no es la exclusividad de datos (postura de EEUU y de la UE) ni la denominada de dependencia permisiva (postura de algunos autores) sino más bien un modelo de “responsabilidad compensatoria" por el cual la información reglamentaria que cumple con los requisitos previos del artículo 39.3 -recientemente citados como presupuestos básicos- no puede ser utilizada por ninguna persona, como tampoco por autoridades regulatorias en su labor de aprobación de nuevos productos, sin que se remunere al titular de los datos ${ }^{14}$. En sentido acorde se ha pronunciado FELLMETH apostando por una remuneración adecuada a fin de que la

\footnotetext{
${ }^{13}$ CORREA, op. cit., pp. 22 y 23.

${ }^{14}$ BASHEER, op. cit., p. 38.
} 
divulgación y el uso de los datos sean justos desde un punto de vista económico ${ }^{15}$. Y MEITINGER revela que el artículo 39.3 requiere que los países miembros garanticen el uso exclusivo de los datos durante un período determinado o que fijen un pago en contraprestación por tal uso ${ }^{16}$.

\section{IMPLICANCIAS DE ADPIC PLUS EN LA FIGURA DE LA EXCLUSIVIDAD DE DATOS}

Tales implicancias dependerán de la interpretación de la norma que en las rondas negociadoras los países miembros de la OMC o firmantes de los respectivos Tratados de Libre Comercio o bilaterales den a la figura. Uno de los mayores temores en la adopción de la figura está en el hecho de que ella acarrearía un aumento en el coste de las medicinas y productos agroquímicos, limitando el acceso a los mismos. El coste se vería aumentado en razón de la misma repetición y práctica en la obtención de los datos de prueba por los laboratorios genéricos, lo cual significa nuevos costos de todo tipo que, en caso de que éstas puedan afrontarlas, repercuten en el precio final del producto ya puesto a la venta. Así, el financiamiento de prolongados ensayos particularmente en países en desarrollo retrasa la actividad y el progreso científico amén de acrecentar el valor del medicamento o del agroquímico, en su caso. Hay que considerar además que la tecnología con la que cuentan y el personal que labora en tales compañías de producción de medicamentos genéricos suele ser limitado y no alcanza el nivel de infraestructura humana o científica ni la capacidad suficiente de soportar financieramente o solventar estudios prolongados, a veces en forma indefinida hasta alcanzar el resultado querido.

Otro, quizás el más sensible, aborda la temática de poner en riesgo la vida y la salud del paciente repitiendo innecesariamente pruebas ya existentes sin justificación científica. Por otro lado, el hecho de que cuánto tiempo puede esperar la sociedad para acceder a medicinas

15 FELLMETH, Aaron. Secrecy, Monopoly, and Acces to Pharmaceuticals in Internacional Trade Law: Protection of Marketing Approval Data under the TRIPS Agreement. Harvard International Law Journal, volumen 45, número 2, 2004, pp. 469-471. Disponible en: <http://www.harvardilj.org/wpcontent/uploads/2011/04/HILJ_45-2_Fellmeth.pdf>. Consultado el: 7 may. 2018.

${ }_{16}$ MEITINGER, Ingo. Implementation of Test Data Protection According to the Article 39.3 TRIPS. The Search for a Fair Interpretation of the Term 'Unfair Commercial Use'. The Journal of World Intellectual Property, volumen 8, número 2, 2005, pp. 125-137. Disponible en: <https://doi.org/10.1111/j.17471796.2005.tb00243.x>. Consultado el: 7 may. 2018. 
genéricas es una pregunta que también comporta un tinte ético donde se encuentra el factor imprescindible de contemplar el acceso a los medicamentos que debe convivir con el del fomento a la innovación de forma equitativa, mas no en detrimento suyo.

No hay que dejar de mencionar que, hasta la figura de las licencias obligatorias, tan necesarias como importantes, pueden verse sensibilizadas ante la presencia de la temática que nos ocupa pues en la negociación de Tratados de Libre Comercio es incierto si el titular de una licencia obligatoria que forma parte de un país firmante de tal tratado puede obtener aprobación para comercializar un producto en caso de que el mismo esté gozando de la protección de exclusividad de datos, salvo que el titular de éstos le conceda la respectiva autorización. Sin ahondar en este tema, cabría también preguntarnos la conveniencia de la actual tendencia en alza de alentar firmas de Tratados multilaterales o de Libre Comercio ya que en la práctica lo que puede ocurrir en temas de acceso a la salud es que el gobierno signatario se vea constreñido a participar de políticas que no le son favorables, viéndose trabadas herramientas que han sido contempladas con éxito por los ADPIC a fin de medir o limitar los monopolios que otorgan los instrumentos de Propiedad Intelectual, como sería el caso de estos permisos que en los países en desarrollo se convierten en la clave para asegurar el acceso a los medicamentos a pacientes que de otra manera no podrían contar con ellos. Resulta un poco contradictorio pensar que un paciente deba estar sujeto a permisos que deban concederse entre titulares: del titular de la licencia obligatoria del medicamento que quedaría supeditado al titular de los datos de prueba del mismo que ejerce una suerte de exclusividad de mercado.

Un caso a resaltar es el de la Comisión Europea, la cual se encuentra reclamando la exclusividad de datos en las negociaciones de su Tratado de Libre Comercio con India y los países andinos a la vez que un reglamento de la propia Comisión establece una excepción de las disposiciones sobre protección de datos en el caso de concederse una licencia obligatoria en un país europeo para exportar medicamentos ${ }^{17}$. Como se aprecia, la discusión sube de tono y adolece de claridad en casos particulares como lo sería la exportación del principio activo titular de la licencia obligatoria.

En cuanto a la agricultura, tintes igualmente profundos se reflejan sobre todo en los países en desarrollo. Un interesante estudio de la Food and Agriculture Organization of the United Nations (FAO), desarrollado por el Panel of Eminent Expets on Ethics in Food and

${ }^{17}$ Correa, op. cit., p. 38. 
Agriculture, Report of Eminent Experts on Ethics in Food and Agriculture que específicamente aborda la figura del data protection y la confidencialidad, revela que en el contexto de los Tratados de Libre Comercio que se celebraron recientemente con algunos países en desarrollo, no es posible basarse en esos datos de prueba, ni utilizarlos por un mínimo de diez años contados a partir de la fecha de aprobación de comercialización del producto, aún si el producto relevante ya no está protegido por la figura de la patente. Esta forma de "exclusividad de datos" limita la competencia y genera precios más elevados para los insumos que los agricultores de los países en desarrollo necesitan, lo cual los excluye de la competencia y les impide producir. Esa exclusividad, en la práctica, puede imponer otra empobrecedora barrera al comercio, tan objetable desde el punto de vista moral como otras barreras que restringen las exportaciones agrícolas de los países empobrecidos. Reducir la pobreza resulta imperativo. Los derechos de Propiedad Intelectual, incluso sobre los datos de prueba, se deben implementar de manera tal que contribuyan a ese objetivo y no para marginar aún más a los agricultores del mundo en desarrollo ${ }^{18}$.

\section{CONCLUSÍON}

Se han visto las diversas dificultades que atraviesa la aplicación de la figura prevista en ADPIC y que comporta la protección de datos de prueba y otros no divulgados y que se relacionan con la seguridad y eficacia en la elaboración de una nueva entidad química que haya significado un esfuerzo considerable para llegar a su obtención. El objetivo principal es evitar prácticas comerciales desleales pero las consecuencias que acarrea su implementación atañen a diversas áreas de la Propiedad Intelectual y también conlleva tintes éticos.

Los procesos de adhesión a acuerdos bilaterales, multilaterales, de libre comercio, con tendencia en alza y que comporta obligaciones para las partes, muchas veces irrenunciables a pesar de la discrecionalidad que prevé igualmente el ADPIC a la hora de adoptar la norma, hacen olvidar a los sujetos negociadores y a los titulares de derechos aspectos imprescindibles de

\footnotetext{
${ }^{18}$ Food and Agriculture Organization of the United Nations (FAO). Panel of Eminent Expets on Ethics in Food and Agriculture. Report of Eminent Experts on Ethics in Food and Agriculture, Third Session, 14-16 September 2005, apartado IV. Disponible en: http://www.fao.org/docrep/010/a0697e/a0697e00.HTM. Consultado el: 7 may. 2018.
} 
considerar, tales como el hecho de que el uso de datos por parte del gobierno no importaría un uso comercial desleal, que existen casos que escapan a la figura por tratarse de utilidad pública o de emergencia sanitaria previstas en la ley a fin de controlar el monopolio en casos específicos (licencias obligatorias), que el costo de la exclusividad de datos en países en vías de desarrollo no puede ser absorbido por los laboratorios de producción local, así como cuestiones referentes a las actuales tendencias que negocian en secrecía los países industrializados.

Además, como se adelantó, hay que tener el cuidado en apuntar que el artículo 39.3 ADPIC deja un espacio considerable a los países miembros para implementar la obligación de proteger datos de prueba, que la información no divulgada se encuentra ya regulada por la competencia desleal en el artículo 10 bis del Convenio de París, así como que la información no divulgada no debe ser abordada dentro del concepto de "propiedad" y no exige conceder derechos exclusivos al titular de los datos.

Hechos estos apuntes, concordamos con la doctrina que sostiene la justa retribución y otorga remuneraciones al titular de los datos por un tiempo determinado, según la ley del país de que se trate y sus justas necesidades y realidades.

Finalmente, referente a la celebración de Acuerdos internacionales, reafirmamos la idea de que una participación pluralista y en igualdad de condiciones en la mesa de negociaciones en el tratamiento de estos temas a nivel internacional podría evitar futuras inconsistencias en la interpretación de la norma, tal como está ocurriendo con el controvertido régimen de concesión de derechos exclusivos sobre datos de prueba en la actualidad, sobre el cual pretendimos ahondar, esclarecer y aportar.

\section{BIBLIOGRAFÍA}

BASHEER, Shamnad. Protection of Regulatory Data Under Article 39.3 of Trips: The Indian Context. Intelectual Property Institute Journal, 2006, p. 11. Disponible en: https: //ssrn.com/abstract=934269. Consultado el: 7 may. 2018.

CORREA, Carlos. Protección de datos de prueba: Derechos conferidos bajo el Acuerdo sobre los ADPIC y algunos efectos de los estándares ADPIC-PLUS en Acceso a los medicamentos e información confidencial. Buenos Aires: Ciudad Argentina-Hispania libros, 2014, pp. 13 y 14. 
FELLMETH, Aaron. Secrecy, Monopoly, and Acces to Pharmaceuticals in Internacional Trade Law: Protection of Marketing Approval Data under the TRIPS Agreement. Harvard International Law Journal, volumen 45, número 2, 2004, pp. 469-471. Disponible en:

<http://www.harvardilj.org/wp-content/uploads/2011/04/HILJ_45-2_Fellmeth.pdf >. Consultado el: 7 may. 2018.

Food and Agriculture Organization of the United Nations (FAO). Panel of Eminent Expets on Ethics in Food and Agriculture. Report of Eminent Experts on Ethics in Food and Agriculture, Third Session, 14-16 September 2005, apartado IV. Disponible en: http://www.fao.org/docrep/010/a0697e/a0697e00.HTM. Consultado el: 7 may. 2018.

MEITINGER, Ingo. Implementation of Test Data Protection According to the Article 39.3 TRIPS. The Search for a Fair Interpretation of the Term 'Unfair Commercial Use'. The Journal of World Intellectual Property, volumen 8, número 2, 2005, pp. 125-137. Disponible en: <https://doi.org/10.1111/j.1747-1796.2005.tb00243.x>. Consultado el: 7 may. 2018.

SATWANT Reddy y GURDIAL Singh Sandhu. Report on Steps to be taken by Government of India in the context of Data Protection Provisions of Article 39.3 of TRIPS Agreement o Informe de India, p. 4. Disponible en:

https://www.indiaoppi.com/sites/default/files/PDF\%20files/Data\%20Protection\%20report.PDF. Consultado el: 7 may. 2018.

Recebido em: 07.05.2018 / Aprovado em: 29.11.2018 / Publicado em: 01.04.2019

\section{COMO FAZER REFERÊNCIA AO ARTIGO (ABNT):}

PEREIRA, Marta Carolina Gimenez. Protección de datos de prueba y su exclusividad en medicamentos y agroquímicos: la interpretación del articulo 39.3 ADPIC. Revista Eletrônica do Curso de Direito da UFSM, Santa Maria, RS, v. 14, n. 1, e32530, jan./abr. 2019. ISSN 1981-3694. DOI: http://dx.doi.org/10.5902/1981369432530. Disponível em: https://periodicos.ufsm.br/revistadireito/article/view/32530 Acesso em: dia mês. ano.

Direitos autorais 2019 Revista Eletrônica do Curso de Direito da UFSM

Editores responsáveis: Rafael Santos de Oliveira e Angela Araujo da Silveira Espindola

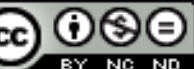

Este obra está licenciado com uma Licença Creative Commons Atribuição-NãoComercial-SemDerivações 4.0 Internacional.

\section{SOBRE A AUTORA}

\section{MARTA CAROLINA GIMÉNEZ PEREIRA}

Possui graduação em Licenciatura en derecho pela Universidad Nacional de Asunción(2002), mestrado em Mestrado em Direito Privado pela Universidad Nacional de Rosario(2004) e doutorado em Doctorado en Derecho pelo Instituto de Investigaciones Jurídicas UNAM(2014). Atualmente é Revisor de periódico da Revista Direito Ambiental e Sociedade, Revisor de periódico da Revista Eletrônica do Curso de Direito da UFSM, Revisor de periódico da CONPEDI LAW REVIEW, Permanente da Universidade Federal da Bahia e Membro de corpo editorial da Revista Opinião Jurídica (FORTALEZA). Tem experiência na área de Direito, com ênfase em Propiedad Intelectual y Nuevas Tecnologías. 